\title{
On Measuring Gravitomagnetism via Spaceborne Clocks: A Gravitomagnetic Clock Effect
}

\author{
B.Mashhoon ${ }^{1,2}$, F.Gronwald ${ }^{2}$, F.W.Hehl ${ }^{2}$, and D.S.Theiss ${ }^{2}$ \\ ${ }^{1}$ Department of Physics and Astronomy \\ University of Missouri, Columbia, Missouri 65211 \\ USA \\ ${ }^{2}$ Institute for Theoretical Physics \\ University of Cologne, 50923 Köln \\ GERMANY
}

\begin{abstract}
The difference in the proper azimuthal periods of revolution of two standard clocks in direct and retrograde orbits about a central rotating mass is proportional to $J / M c^{2}$, where $J$ and $M$ are, respectively, the proper angular momentum and mass of the source. In connection with this gravitomagnetic clock effect, we explore the possibility of using spaceborne standard clocks for detecting the gravitomagnetic field of the Earth. It is shown that this approach to the measurement of the gravitomagnetic field is, in a certain sense, theoretically equivalent to the Gravity Probe-B concept.
\end{abstract}




\section{Introduction}

Currently there is considerable interest in the development of highly stable clocks for space applications [1]. This circumstance provides the impetus to investigate further a certain remarkable gravitomagnetic clock effect [2] within the framework of general relativity.

The Newtonian theory of gravitation may be thought of as the nonrelativistic theory of the gravitoelectric field $\mathbf{E}_{g}$, while general relativity involves - among other things the gravitomagnetic field $\mathbf{B}_{g}$ as well. These notions are ultimately based on the close formal analogy between Newton's law of universal gravitation and Coulomb's law of electricity. The gravitomagnetic clock effect involves a certain characteristic temporal structure around rotating bodies. The elucidation of the various aspects of this effect is the main subject of this paper; in particular, we consider the theoretical problem of measuring the non-Newtonian gravitomagnetic field by means of ultra-stable clocks in space.

The standard tests of the Einstein theory of gravitation can be accounted for by postNewtonian gravitoelectric corrections: gravitational redshift, perihelion precession of Mercury, bending of light in the field of the Sun, and Shapiro's radar time delay. The investigation of gravitomagnetic effects in general relativity began with the work of de Sitter, Thirring, and Lense and Thirring [3]. However, the idea of a gravitomagnetic field generated by mass current dates back to the last century when developments in electromagnetism suggested a generalization of Newton's theory of gravitation along the lines of electrodynamics [4, 5]. Holzmüller [6] and Tisserand [0] tried to explain the perihelion excess of Mercury by taking into account solar gravitomagnetism. The analogy with electrodynamics has been the subject of many studies [8, 9, 10]; in fact, any theory that brings together Newtonian gravitation and Lorentz invariance must contain gravitomagnetism in some form [11]. At present, general relativity is consistent with all observational data and within its framework the theoretical development of gravitoelectromagnetism has reached a certain level of maturity [12]. On the experimental side, moreover, the main mission of NASA's Gravity Probe-B will be the direct measurement of the gravitomagnetic field of the Earth via superconducting gyroscopes carried by a drag-free satellite in a polar orbit about the Earth [13].

In this paper, we choose an astronomical body such as the Earth for the sake of concreteness and concentrate our attention on the theoretical possibilities for measuring its gravitomagnetism by means of spaceborne clocks. To this end, the exterior gravitational field of the Earth may be considered in the linear approximation with a metric of the form

$$
-d s^{2}=-c^{2}\left(1-\frac{2 G M}{c^{2} \rho}\right) d t^{2}+\left(1+\frac{2 G M}{c^{2} \rho}\right) \delta_{i j} d x^{i} d x^{j}-\frac{4 G d t}{c^{2} \rho^{3}} \epsilon_{i j k} J^{i} x^{j} d x^{k},
$$

where $M$ and $J$ are the mass and angular momentum of the body, respectively. Here $\rho$ is the isotropic radial coordinate in an underlying Cartesian coordinate system $\boldsymbol{\rho}=$ 
$\left(x^{i}\right)=(x, y, z)$ and we choose an orientation such that $\mathbf{J}=J \hat{\mathbf{z}}$. The Lense-Thirring part of this metric has the form $-4 c^{-1}\left(\mathbf{A}_{g} \cdot d \mathbf{x}\right) d t$, where

$$
\mathbf{A}_{g}=\frac{G}{c} \frac{\mathbf{J} \times \boldsymbol{\rho}}{\rho^{3}}
$$

is the gravitomagnetic vector potential. A free ideal gyroscope held at rest at a fixed position $\boldsymbol{\rho}$ in space would precess in the gravitomagnetic field at a rate given by

$$
\boldsymbol{\Omega}_{P}=\frac{G}{c^{2} \rho^{5}}\left[3(\mathbf{J} \cdot \boldsymbol{\rho}) \boldsymbol{\rho}-\rho^{2} \mathbf{J}\right]
$$

in this approximation. The gravitomagnetic field is then defined by $\mathbf{B}_{g}=c \boldsymbol{\Omega}_{P}=\nabla \times \mathbf{A}_{g}$, while the gravitoelectric field is $\mathbf{E}_{g}=-\nabla \Phi_{N}$, where $\Phi_{N}=G M / \rho$ is the Newtonian potential, as expected.

In the following sections 2 and 4, the Schwarzschild radial coordinate $r$ is employed,

$$
r=\rho\left(1+\frac{1}{2} \frac{G M}{c^{2} \rho}\right)^{2}
$$

moreover, we usually set $G=1$ and $c=1$ for the sake of simplicity - except where indicated otherwise. The gravitomagnetic clock effect under consideration in this paper is described in terms of azimuthal closure for simple geodesic orbits in sections $2-4$. This gravitomagnetic effect may be thought of in terms of a certain limiting form of the gravitational Aharonov-Bohm effect; indeed, this connection leads to a discussion of holonomy in section 5. A preliminary examination of the experimental possibilities is provided in section 6 . The measurement of the gravitomagnetic clock effect appears to be beyond present experimental capabilities by almost an order of magnitude.

\section{A Gravitomagnetic Clock Effect}

A standard clock by definition measures proper time along its worldline. Imagine such clocks in the stationary spacetime outside a charged rotating source; in fact, we take this region to be the exterior Kerr-Newman spacetime for the sake of simplicity.

Consider equatorial circular geodesic orbits in this spacetime. We are interested in timelike orbits that are stable against radial perturbations. This is possible for circular orbits only beyond a certain radius. Let $t_{+}\left(t_{-}\right)$be the period of revolution in the same (opposite) sense as the rotation of the source for the stable circular geodesic path with fixed radial coordinate $r$ in standard Schwarzschild-like coordinates. It can be shown that

$$
t_{ \pm}=T_{0} \pm 2 \pi \frac{a}{c}
$$


where $T_{0}=2 \pi / \omega_{0}, \omega_{0}$ is the modified "Keplerian" frequency given by

$$
\omega_{0}=\left(\frac{G M}{r^{3}}-\frac{G Q^{2}}{c^{2} r^{4}}\right)^{\frac{1}{2}}
$$

$Q$ denotes the charge, and $a=J / M c$ is the Kerr parameter 14. The coordinate time $t$ is the proper time of static asymptotically inertial observers that are infinitely far from the source. It follows from equation (5) that

$$
t_{+}-t_{-}=4 \pi \frac{J}{M c^{2}} .
$$

The operational significance of this interesting result is doubtful, since light signals are required, for instance, to carry information about the orbit to the distant clocks. To ameliorate this situation, let us consider instead the proper periods, $\tau_{ \pm}$, of such circular paths measured by free orbiting standard clocks. It is possible to show that 14

$$
\tau_{ \pm}=T_{0}\left[1-\frac{3 G M}{c^{2} r}+2 \frac{G Q^{2}}{c^{4} r^{2}} \pm 2 \frac{a}{c} \omega_{o}\right]^{\frac{1}{2}}
$$

so that

$$
\frac{1}{2 T_{0}}\left(\tau_{+}^{2}-\tau_{-}^{2}\right)=4 \pi \frac{J}{M c^{2}}
$$

hence, an experimental determination of the left side of equation (9) would lead to the measurement of $J / M$ for the source. Specifically, let us write equation (9) as

$$
\tau_{+}-\tau_{-}=4 \pi \frac{J}{M c^{2}} U(r)
$$

where $U^{-1} \equiv \frac{1}{2}\left(\tau_{+}+\tau_{-}\right) / T_{0}$ and $U(r)$ approaches unity as $r \rightarrow \infty$. Under physically realistic conditions, $U$ is a monotonically decreasing function of $r$; in fact, for

$$
\Phi \equiv \frac{G M}{c^{2} r} \ll 1
$$

$U(r)$ has an expansion of the form

$$
U(r)=1+\frac{3}{2} \Phi+\left(\frac{27}{8}-\frac{Q^{2}}{G M^{2}}\right) \Phi^{2}+\left(\frac{135}{16}+\frac{1}{2} \frac{c^{2} J^{2}}{G^{2} M^{4}}-\frac{9}{2} \frac{Q^{2}}{G M^{2}}\right) \Phi^{3}+\cdots
$$

The exact result (8) is valid for the Kerr-Newman geometry. We are interested, however, in the exterior field of a rotating astronomical body; therefore, $r \gg G M / c^{2}$ and hence

$$
\tau_{+}-\tau_{-} \simeq 4 \pi \frac{J}{M c^{2}}
$$

This is a remarkable relation that could, in principle, be used to measure $J / M$ directly for an astronomical body; in fact, for the Earth $\tau_{+}-\tau_{-} \simeq 2 \times 10^{-7} \mathrm{sec}$, while for the Sun 
$\tau_{+}-\tau_{-} \simeq 10^{-5}$ sec. Equations (7) and (13) indicate a general feature of time in the field of a rotating source; this paper is devoted to an account of this gravitomagnetic clock effect and its possible observational significance.

The general result that it takes longer for a free test particle to go around a rotating mass in its equatorial plane in the prograde direction than in the retrograde direction is a remarkable fact that is in conflict with the notion that a rotating mass drags space around with it; in fact, such a "Machian" concept is in conflict with general relativity and must be abandoned [3, 15]. Moreover, the "dragging of local inertial frames" has often been used as a metaphor for the gravitomagnetic precession of ideal test gyroscopes; however, it must be remarked that even this figurative usage has the erroneous connotation just mentioned. There are two other interrelated aspects of the general relation (13) that are quite interesting and require further discussion. First, it is clear from equation (13) that $\tau_{+}-\tau_{-}$is nearly independent of Newton's gravitational constant $G$. Intuitively, this essentially comes about since in this approximation a "small" quantity is integrated over a "long" interval. Thus the result could be a "large" effect, since it is independent of the extremely weak gravitational coupling constant in this limit. It is useful to recall here a similar situation involving the total gravitational radiation energy emitted when a test particle of mass $m$ that is at rest at infinity falls radially into a Schwarzschild black hole of mass $M \gg m$; the net result, $\simeq 10^{-2}(\mathrm{~m} / M) m c^{2}$, is also independent of $G$. Another analogous circumstance involves the net amplitude of relativistic nutation that is independent of $c$; that is, the spin vector of a test particle in an orbit of inclination $\alpha$ about a rotating mass undergoes - in addition to the normal precessional motions - a certain nutational motion of long (Fokker) period $T_{F} \simeq 2 T_{0} / 3 \Phi$ with an amplitude $J \sin \alpha / M r^{2} \omega_{0}$ that is independent of $c$ [16]. This is a consequence of the post-Schwarzschild approximation scheme that is discussed in section 4 .

The other significant aspect of the result (13) is that it is essentially independent of $r$ for $r \gg 2 G M / c^{2}$. This gravitomagnetic clock effect is thus reminiscent of the topological Aharonov-Bohm effect. The connection between these effects can be further clarified as follows: The Aharonov-Bohm effect is simply related to the Sagnac effect via the Larmor theorem [17]; in a similar way, the gravitational Larmor theorem provides a connection between the gravitomagnetic effect (13) and an analog of the Sagnac effect. This is discussed in the next section.

\section{Analogy with the Sagnac Effect}

A century ago, Larmor's theorem provided a local connection between magnetism and rotation. The theorem applies to circumstances involving slowly moving charged particles and slowly varying fields whose strengths are considered only to first order. It turns out (cf. Appendix A) that the theorem can be extended to the gravitational case such that the gravitoelectric charge of a test mass $m$ would be $q_{E}=-m$ and its gravitomagnetic charge would be $q_{B}=-2 m$. This is the content of the gravitational Larmor 
theorem [18]; the negative signs of the gravitational charges account for the attraction of gravity and $q_{B} / q_{E}=2$ since gravity is a spin-2 field.

It follows from Larmor's relation

$$
\boldsymbol{\Omega}_{L}=\frac{q}{2 m c} \mathbf{B}
$$

that in the gravitomagnetic case with $q_{B}=-2 m$, we have

$$
\Omega_{L}=-\Omega_{P}
$$

where $\boldsymbol{\Omega}_{P}=\mathbf{B}_{g} / c$ is the local gravitomagnetic precession frequency of a gyroscope at rest. It follows from equation (3) that in the equatorial plane $(\mathbf{J} \cdot \boldsymbol{\rho}=0)$ one should obtain the same physical result at an orbit of radius $\rho$ in the absence of gravity but in a frame rotating with uniform frequency $\Omega_{L}=G \mathbf{J} / c^{2} \rho^{3}$. Imagine, therefore, two clocks moving in opposite directions with speed $v$ on the circular orbit of radius $\rho$ in the absence of gravity. According to an observer at rest in the frame rotating with frequency $\Omega_{L}$, the periods of circular motion for the two clocks are

$$
t_{ \pm}=\frac{2 \pi \rho}{v \mp \rho \Omega_{L}}
$$

by the nonrelativistic law for the addition of velocities. (The same result can be obtained with respect to static observers in the underlying inertial frame.) It follows that

$$
t_{+}-t_{-}=\frac{4\left(\pi \rho^{2}\right) \Omega_{L}}{v^{2}-\rho^{2} \Omega_{L}^{2}},
$$

which for $v \gg \rho \Omega_{L}$ and $v \rightarrow c$ reduces to the Sagnac effect for light - though the derivation presented here breaks down, of course, as it is only valid in the nonrelativistic approximation. That is, the Sagnac phase shift may be thought of - in the eikonal approximation - as the product of frequency of light and the total time difference for light to traverse the circular area in opposite directions. As is well known, the Sagnac effect is proportional to the enclosed area $\left(\pi \rho^{2}\right)$; in fact, the magnitude of the Sagnac phase shift is given in our case by $4 \omega^{*}\left(\pi \rho^{2}\right) \Omega_{L} / c^{2}$, where $\omega^{*}$ is the frequency of the electromagnetic radiation.

Let us now compute equation (17) for the gravitomagnetic case $v=\rho \omega_{0}$, where $\omega_{0}$ is the Keplerian frequency, $\omega_{0}^{2}=G M / \rho^{3}$, and

$$
\frac{\Omega_{L}^{2}}{\omega_{0}^{2}}=\frac{J}{M c^{2}} \Omega_{L} \ll 1
$$

in all physically realistic situations. Hence we find that

$$
\tau_{+}-\tau_{-} \simeq 4 \pi \frac{\Omega_{L}}{\omega_{0}^{2}}=4 \pi \frac{J}{M c^{2}},
$$


as expected from the gravitational Larmor theorem.

The simplicity of the above argument is due to the fact that at a given $\rho$ in the equatorial plane $\Omega_{L}$ is uniform; however, for an orbit at an inclination $\alpha \neq 0$ this would no longer be the case. A more significant problem is that an orbit in the field of a rotating mass is not in general spatially closed. In fact, the equatorial circular geodesic orbits discussed thus far are exceptional in this respect. It is therefore necessary to formulate a generalization of relation (13) for an arbitrary orbit; this problem is treated in the next section.

\section{Standard Clocks in Space}

A spaceborne clock would in general follow a complicated orbit. Thus far, we have considered only circular geodesic orbits in the equatorial plane of a rotating mass; however, it is possible to generalize the gravitomagnetic clock effect to the case of an arbitrary orbit. To clarify the situation, we first consider a "spherical" orbit that has a small inclination $\alpha \ll 1$ with respect to the equatorial plane. Spherical orbits in Kerr spacetime have been described by Wilkins [19]. Such a geodesic orbit is no longer spatially closed in general; therefore, it is necessary to define the relevant periods $\tau_{ \pm}$in terms of azimuthal closure.

Most elementary astronomical systems, i.e. stars and planets, are nearly spherical bodies. The exterior gravitational field of a spherically symmetric mass distribution is uniquely described by the Schwarzschild spacetime; therefore, it is natural to express the exterior field of such an astronomical system in terms of perturbations of the Schwarzschild field. The post-Schwarzschild approximation scheme is a useful method in relativistic celestial mechanics [16, 20, 21]. It is possible, in principle, to include the quadrupole and higher mass moments of the source in our treatment; however, the analysis of the gravitomagnetic clock effect for a general orbit would then become much more complicated and is beyond the scope of this paper. The unique first-order angular momentum perturbation is simply given by the Kerr field linearized in the angular momentum parameter $a$. Thus we express the exterior field of a rotating mass by

$$
-d s^{2}=-c^{2}(1-2 \Phi) d t^{2}+(1-2 \Phi)^{-1} d r^{2}+r^{2}\left(d \theta^{2}+\sin ^{2} \theta d \varphi^{2}\right)-4 c r \Psi \sin ^{2} \theta d t d \varphi,
$$

where $\Psi=G J / c^{3} r^{2}$ is a gravitomagnetic potential. For the exterior field of the Earth, $\Phi(r)<7 \times 10^{-10}$ and $\Psi(r)<4 \times 10^{-16}$.

Let us now consider a "spherical" orbit of small inclination $\alpha \ll 1$ given by

$$
\begin{aligned}
t & =\Gamma_{0}\left(1-3 \frac{a \omega}{c} \Gamma_{0} \Phi_{0}\right) \tau \\
r & =r_{0}, \\
\theta & =\frac{\pi}{2}-\alpha \sin \eta+3 \alpha \frac{a \omega}{c} \Gamma_{0}\left(1-2 \Phi_{0}\right) \omega \tau \cos \eta, \\
\varphi & =\left(1-\frac{a \omega}{c} \Gamma_{0}\right) \omega \tau+\varphi_{0},
\end{aligned}
$$


where $\Phi_{0}=\Phi\left(r_{0}\right), \omega$ is the proper frequency of the unperturbed orbit, $\omega=\omega_{0} \Gamma_{0}$,

$$
\Gamma_{0}=\left(1-3 \Phi_{0}\right)^{-\frac{1}{2}}
$$

and $\eta$ is - for the unperturbed orbit - the phase angle in the orbital plane measured from the line of nodes

$$
\eta=\omega \tau+\eta_{0}
$$

The constants $\varphi_{0}$ and $\eta_{0}$ are given in terms of initial conditions at $\tau=0$ as in Figure 1. The linear perturbation analysis is valid for $\omega_{0} \tau \ll\left(a \omega_{0} / c\right)^{-1}$. It is possible to show that the average behavior of the orbit is described by the Lense-Thirring effect with a precession frequency $\Omega_{L T} \simeq 2 c \Psi\left(r_{0}\right) / r_{0}$.

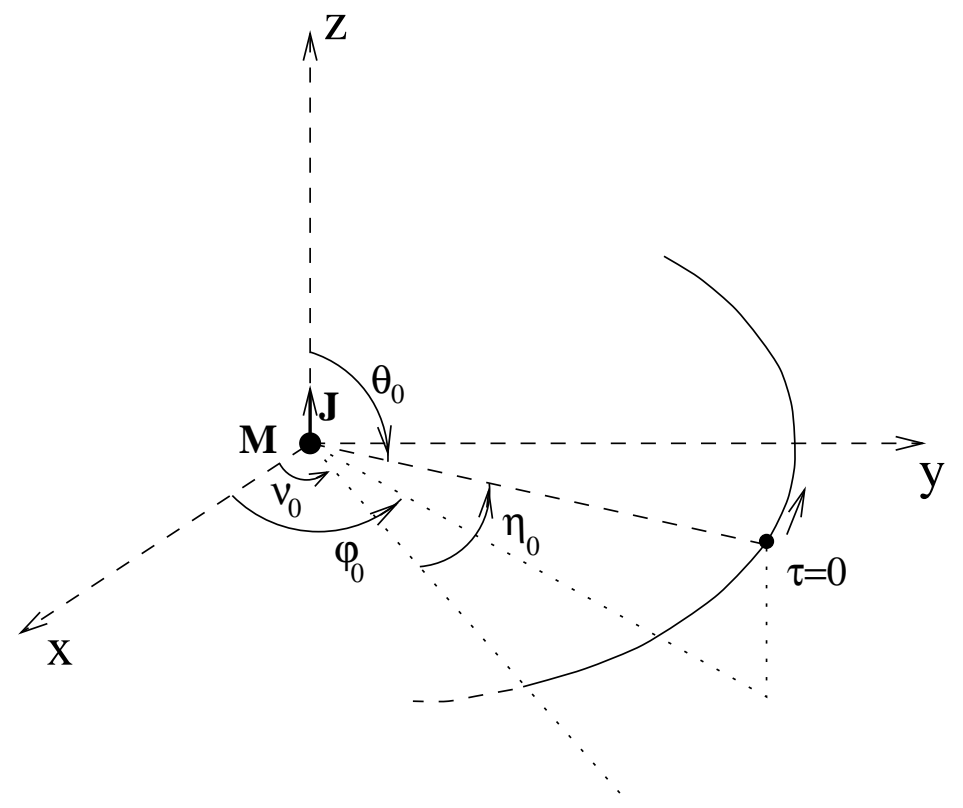

Figure 1: Plot of the direct "spherical" orbit of radius $r_{0}$. The azimuth of the line of nodes $\nu_{0}$ is given by $\nu_{0}=\varphi_{o}-\arctan \left(\cos \alpha \tan \eta_{0}\right)$. The polar angle at $\tau=0$ is given by $\cos \theta_{0}=\sin \alpha \sin \eta_{0}$. Here $\alpha$ is the inclination of the unperturbed (circular) orbit with respect to the equatorial plane of the rotating mass.

It is clear from equations (21) - (24) that the orbit is no longer spatially closed once $\alpha \neq 0$. Let us define $\tau_{+}$to be the period of motion from $\varphi_{0}$ to $\varphi_{0}+2 \pi$; hence, we obtain from equation (24)

$$
\left(1-\frac{a \omega}{c} \Gamma_{0}\right) \omega \tau_{+}=2 \pi .
$$

It follows from this relation that $(T=2 \pi / \omega)$

$$
\tau_{+}=T+2 \pi \Gamma_{0} \frac{a}{c}
$$


moreover, for a retrograde orbit, $a \rightarrow-a$ so that

$$
\tau_{-}=T-2 \pi \Gamma_{0} \frac{a}{c} .
$$

Hence, we recover $\tau_{+}-\tau_{-} \simeq 4 \pi a / c$ for $\Phi_{0} \ll 1$. Thus the rotation of the source breaks the degeneracy in the proper period $T$ of the orbit by an amount that is of the order of $J / M c^{2}$. This is illustrated in Figure 2.

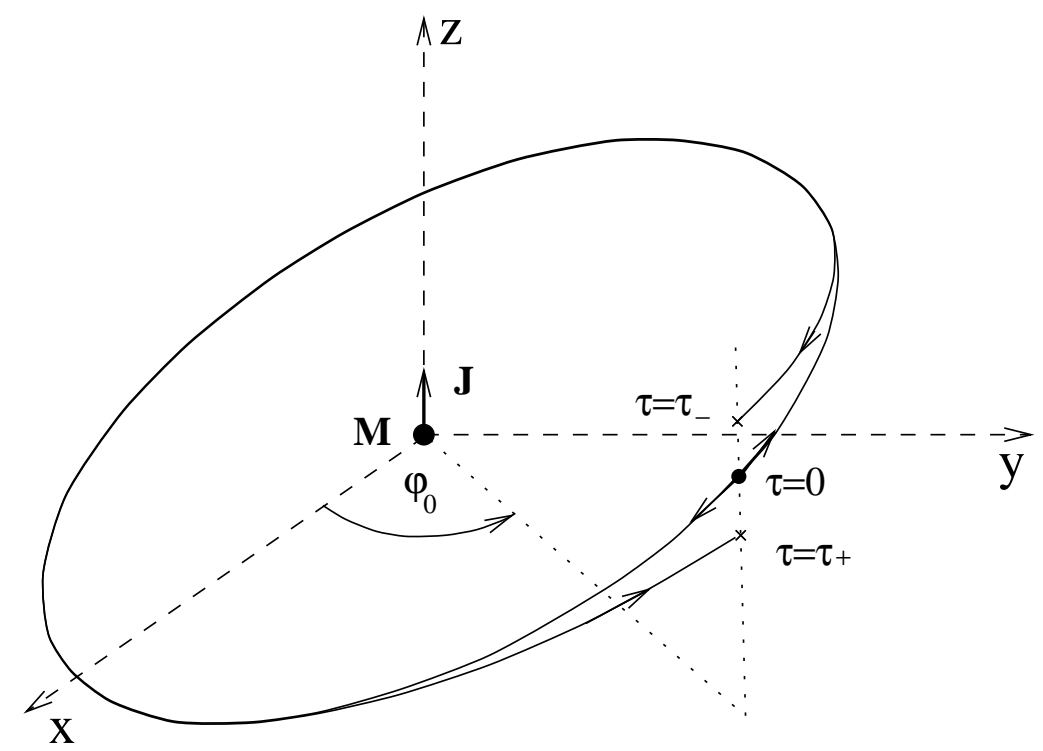

Figure 2: Schematic plot of clock orbits about a rotating mass. The azimuthal periods are illustrated for $\cos \eta_{0}>0$; in fact, the deviation in the polar angle after one orbit is $4 \pi \alpha a \cos \eta_{0} / c \Gamma_{0}$.

Let us next consider a general "spherical" orbit of arbitrary inclination $\alpha$; in fact, the orbital equations have been derived in previous work 20]. We have

$$
\begin{aligned}
t(\tau) & =\Gamma_{0}\left(1-3 \frac{a \omega}{c} \Gamma_{0} \Phi_{0} \cos \alpha\right) \tau, \\
r(\tau) & =r_{0}, \\
\theta(\tau) & =\arccos (\sin \alpha \sin \eta)+\frac{3}{2} \frac{a \omega}{c} \Gamma_{0}\left(1-2 \Phi_{0}\right) \sin (2 \alpha) \frac{\cos \eta}{\sigma(\eta)} \omega \tau, \\
\varphi(\tau) & =\arctan (\cos \alpha \tan \eta)+\frac{a \omega}{c}\left[2 \Gamma_{0}^{-1}-3 \Gamma_{0}\left(1-2 \Phi_{0}\right) \frac{\cos ^{2} \alpha}{\sigma^{2}(\eta)}\right] \omega \tau+\nu_{0},
\end{aligned}
$$

where $\eta$ is defined as in equation (26), $\sigma(\eta)$ is given by

$$
\sigma(\eta)=\left(1-\sin ^{2} \alpha \sin ^{2} \eta\right)^{\frac{1}{2}}
$$


and $\nu_{0}$ is the longitude of the node as in Figure 1. For $\alpha \ll 1$, equations $(30)-(33)$ reduce to the previous orbital equations (21) - (24); moreover, the Lense-Thirring precession as well as other properties of the general orbit have been described in detail before [20]. For $\alpha=\pi / 2$, the main orbital motion is polar and the azimuthal motion indicates the Lense-Thirring precession of the nodes; therefore, the gravitomagnetic clock effect is absent in this case. Hence we assume $\alpha \neq \pi / 2$ and consider $\tau_{+}$from equation (33) such that $\varphi_{0} \rightarrow \varphi_{0}+2 \pi$ from $\tau=0$ to $\tau=\tau_{+}$; we find an implicit equation for $\tau_{+}$that can be solved perturbatively. Thus let

$$
\tau_{ \pm}=T \pm 2 \pi \frac{a}{c} \lambda \cos \alpha
$$

then, it is possible to show that

$$
\lambda=\Gamma_{0}-2 \Gamma_{0}^{-1} \tan ^{2} \alpha \cos ^{2} \eta_{0}
$$

for $\alpha$ sufficiently different from $\pi / 2$ such that the perturbative treatment remains valid. It is important to recognize that $\lambda$ could change sign and become negative for sufficiently large inclination of the orbital plane with respect to the equatorial plane of the central source. For instance, for $\alpha$ and $\eta_{0}$ both near $\pi / 4, \lambda$ could go through zero and change sign. The azimuthal period of the prograde orbit would then become shorter than the retrograde orbit.

The influence of the gravitomagnetic field of the source is in general reflected in any orbital timing mechanism. For instance, let us recall that $t$ is the proper time of static observers at spatial infinity. Thus if the orbital motion about a rotating source is referred to static clocks at spatial infinity, we find

$$
t_{ \pm}=T_{0} \pm 2 \pi \frac{a}{c} \Gamma_{0} \lambda^{\prime} \cos \alpha
$$

where $\Gamma_{0} T=T_{0}$ and $\lambda^{\prime}=\lambda-3 \Phi_{0} \Gamma_{0}$ using equations (30) and (35). This consideration could possibly be useful in the experimental determination of $J / M$ as well.

It follows from these considerations that in the approximation under consideration, where the rotation of the source is taken into account only to first order, the azimuthal orbital period $\tau$ - as compared to the proper Keplerian period $T$ - would be larger (smaller) due to orbital motion with $\lambda>0$ in the same (opposite) sense as the rotation of the source. For the case of a satellite around the Earth, this difference is $\pm 2 \pi a \lambda \cos \alpha / c$, where $2 \pi a / c \approx 10^{-7}$ sec. A similar situation holds - as noted in the previous paragraph even when the binary system is far away. It is interesting to contrast this circumstance with the variation in the period of the Hulse-Taylor binary pulsar PSR B1913+16. The binary period is monotonically decreasing by about $10^{-7}$ sec per orbit as a result of gravitational radiation damping, since the observations agree with the theoretically estimated decay due to the emission of gravitational radiation by the binary system. To measure an effect of this size for the motion of a clock in orbit around the Earth is the observational challenge posed by the gravitomagnetic clock effect (cf. section 6). 
Though our approach has been macrophysical throughout, it is nevertheless interesting to note that for gravitational orbits around a neutron, say, $\tau_{+}-\tau_{-}$is equal to the Compton period of the neutron.

Finally, imagine standard clocks in orbit about a static astronomical source. The orbital period is defined by azimuthal closure as before. Suppose that for two clocks starting at $\tau=0$ in opposite directions around the source we have $\tau_{+}=\tau_{-}$. This degeneracy is removed once the source rotates [cf. equation (35)]. It is then expected that $\tau_{+}-\tau_{-}=$ $4 \pi a \lambda \cos \alpha / c$ would be the dominant relativistic rotation-dependent term in general.

\section{Holonomy}

As already mentioned in section 2, the gravitomagnetic clock effect can be considered as a gravitational analog of the Aharonov-Bohm effect. It is well known that the AharonovBohm effect is closely connected to the concept of holonomy (see, for example, [22], section 10.5.3.): The phase shift observed in the Aharonov-Bohm experiment can be obtained after the integration of $U(1)$-parallel transport around a closed loop which surrounds a magnetic field. Mathematically, this phase shift is given by the holonomy attributed to a fibre bundle with base space $S^{1}$ (representing the closed loop), fibre $U(1)$, and a $U(1)$-valued connection (the vector potential that corresponds to the magnetic field). An analogous construction can also be conceived in order to obtain the gravitomagnetic clock effect as a translational holonomy of a fibre bundle. In this case one defines a fibre bundle with base space $S^{1}$ (representing the parameter space of the azimuthal coordinate $\varphi$ ), the real line $R$ as fibre, and an $R$-valued connection (describing the time shift of an orbiting clock due to gravitomagnetism). However, this construction is of limited use since the holonomy obtained is not directly related to gravitational holonomy.

Gravitational holonomy is a characteristic of exterior parallel transport on spacetime. More precisely, it is attributed to an affine frame bundle which takes spacetime as base manifold and is equipped with an affine connection (cf. Appendix B). The gravitational holonomy itself splits into translational holonomy and rotational holonomy. In the limit of infinitesimally closed and contractible loops these holonomies turn, respectively, into the torsion and curvature of spacetime. In general relativity, one is restricted to Riemannian parallel transport such that torsion vanishes and curvature becomes Riemannian curvature. However, it should be noted that, in general, Riemannian parallel transport on a Riemannian manifold exhibits nonvanishing translational holonomy. This circumstance does not imply the presence of nontrivial torsion, of course. Nevertheless, Petti [23] employed spacelike circular curves in the Kerr geometry in a significant attempt to relate the angular momentum that occurs in the Kerr metric to torsion via translational holonomy. Indeed, it was outlined in [23] how to assemble a manifold with torsion from torsion-less Kerr configurations in a manner similar to the assembly of a simplicial Riemannian manifold from piecewise flat spaces. This interesting undertaking deserves 
further investigation since - as Petti [23] has observed - a careful limiting process should be taken into account.

It follows from this that the gravitomagnetic clock effect does not give us any information on the local geometry of the Kerr metric that goes beyond Riemannian geometry. This is not really surprising since an ideal standard clock, if considered as a structureless, i.e. pointlike, test mass, does not couple to torsion at all [24]; in fact, it measures proper time along timelike worldlines and is thus intimately tied to the metric concept of Riemannian geometry.

\section{Discussion}

NASA's Gravity Probe-A involved the sub-orbital flight of a rocket carrying a hydrogen maser clock; the main result of this experiment was an accurate test of the gravitational redshift by Vessot et al. [25, 26]. It is expected that some of the future space missions will carry ultra-stable clocks, and hence it is possible that the gravitomagnetic effect discussed in this paper could be measurable in the future. For an orbit of Keplerian period $T_{0}$, equations (35) and (36) imply that the relative gravitomagnetic variation in orbital period is

$$
\frac{\tau_{+}-\tau_{-}}{T_{0}} \simeq \frac{2 J}{M c^{2}}\left(\frac{G M}{\rho^{3}}\right)^{1 / 2}
$$

or about $4 \times 10^{-11}$ for a near-Earth orbit.

The principal aim of NASA's Gravity Probe-B will be the direct measurement of the gravitomagnetic field of the Earth by comparing the precession of gyroscopes with respect to telescopes on board a drag-free satellite in a polar orbit about the Earth [13]. After one orbital period, the gravitomagnetic precession angle of the gyroscope per $2 \pi$ radians is

$$
\frac{\Omega_{P}}{\omega_{0}} \simeq \frac{2 G J}{c^{2} \rho^{3}}\left(\frac{G M}{\rho^{3}}\right)^{-1 / 2},
$$

which is identical with equation (38). This equality indicates that the theoretical possibility of measuring gravitomagnetism via clocks is essentially equivalent to the GP-B.

The gravitomagnetic clock effect constitutes a surprisingly large effect. We mentioned already in section 2 that in the case of an astronomical body such as the Earth, the time difference $\tau_{+}-\tau_{-} \simeq 4 \pi J / M c^{2}$ after one orbit amounts to

$$
\tau_{+}-\tau_{-} \simeq 2 \times 10^{-7} \mathrm{sec}
$$

Time shifts due to the gravitomagnetic field of the Earth are widely believed to be of much lower order. Indeed, suppose the time difference of a direct and retrograde moving 
clock is taken at a fixed time, say, after one Kepler period $T_{0}=2 \pi / \omega_{0}$. Then it is straightforward to show that for a circular equatorial orbit,

$$
\tau_{+}^{\prime}-\tau_{-}^{\prime}=12 \pi \frac{G J}{c^{4} r}+\mathcal{O}\left(c^{-6}\right)
$$

As an example, we set $r=7000 \mathrm{~km}$ and obtain

$$
\tau_{+}^{\prime}-\tau_{-}^{\prime} \simeq 3 \times 10^{-16} \mathrm{sec}
$$

This is nine orders of magnitude smaller than the time difference (40). We recall that the result (40) presupposes that the time difference of the two clocks is taken with respect to a fixed angle $\varphi$ (i.e., after each clock has covered an azimuthal interval of $2 \pi$ ) and not with respect to a fixed time.

In principle, it is a trivial task to measure a time difference of $2 \times 10^{-7}$ sec with today's technology. However, an experimental verification of the gravitomagnetic clock effect does not only require the measurement of the time difference between two well-defined events up to an accuracy of $2 \times 10^{-7} \mathrm{sec}$. It is, in fact, the proper time along the direct and retrograde orbits that is used as a clock. Therefore, it is not sufficient to send two highly accurate and stable clocks into space and let them orbit in opposite directions. The orbits themselves have to be highly accurate and stable as well. We recall that in order to obtain the time difference (40) we have to subtract two periods, each of which represents the sum of a Kepler period and a much smaller relativistic correction. Under the assumption of identical orbits, the Kepler periods and their gravitoelectric corrections cancel upon subtraction while the gravitomagnetic contributions add up, yielding the actual clock effect under consideration here. Disturbances of the orbits will in general change the Kepler periods of the orbiting clocks. It follows that in this case the Kepler periods will not exactly cancel but may exhibit a significant time difference.

In order to obtain some estimation of possible errors, we consider an actual experiment where atomic clocks are on board satellites in direct and retrograde orbits around the Earth. We may divide the error sources of such an experiment into two groups, namely

(i) errors due to the tracking of the actual orbits, and

(ii) deviations from idealized orbits due to

- mass multipole moments of the Earth

- radiation pressure

- gravitational influence of the Moon, the Sun, and other planets

- other systematic errors (e.g., atmospheric disturbances).

The tracking of the actual orbits requires the measurement of distances and angles. The position of a satellite along an orbit can be determined to a few centimeters using the Global Positioning System (GPS); therefore, the temporal uncertainty that a nearEarth satellite has actually returned to the same azimuthal position in space is about $\delta \tau \approx \delta r / v \approx 10^{-6}$ sec. Here $\delta r \approx 1 \mathrm{~cm}$ is the position uncertainty along track and $v$ 
is the orbital speed of the satellite. The gravitomagnetic clock effect, however, involves a definite temporal deviation of $\sim 10^{-7}$ sec. It turns out that one should be able to measure the orbital radius up to an accuracy of the order of $10^{-2} \mathrm{~cm}$ and to determine angles up to an accuracy of $10^{-10} \mathrm{rad}$ in order to keep the errors due to the measurement smaller than the clock effect after one orbit. These requirements are about one order of magnitude higher than what can be achieved today. On the other hand, the clock effect is cumulative - just like the precession angle of a GP-B gyroscope - and hence many orbital periods can be used for a measurement of the gravitomagnetic effect; that is, the statistical tracking errors could be overcome if one were able to perform many single measurements [27].

The astrometric requirements for ensuring azimuthal closure should be emphasized. The operational definition of the azimuthal angle $\varphi$ is ultimately based on the underlying astronomical coordinate system employed [28]. In fact, it may turn out to be necessary to monitor orbital motion so accurately as to be able to measure the Lense-Thirring effect and hence gravitomagnetism directly using only astrometric data on the orbits of spaceborne clocks.

The systematic errors of the second group of error sources have a more serious influence on the gravitomagnetic clock effect. In order to calculate the influence of such perturbative accelerations on the Kepler period one has to focus on sections of orbits rather than on complete closed orbits. This is because under favorable conditions (e.g., an almost constant perturbative acceleration) different temporal deviations can cancel each other when summed over a closed orbit. It is possible to estimate that perturbative accelerations should be kept below $10^{-11} \mathrm{~g}$ in order for the clock effect to become measurable.

Perturbative accelerations due to multipole moments of the Earth are of the order of $10^{-3} \mathrm{~g}$. The orbit of a satellite under the influence of the nonspherical and inhomogeneous form of the Earth may be likened to a bumpy road. However, we do know the gravitational field of the Earth up to an accuracy of $10^{-9} \mathrm{~g}-10^{-10} \mathrm{~g}$. NASA's already approved gravity mapping mission GRACE is expected to push this accuracy higher by about two orders of magnitude [29]. This would then make it possible, in principle, to correct for the influence of the multipole moments of the Earth on a gravitomagnetic clock experiment.

The radiation pressure of the Sun causes perturbative accelerations of the order of $10^{-8} \mathrm{~g}$. Using drag-free satellite techniques, this disturbance can be reduced by two orders of magnitude to $10^{-10} \mathrm{~g}$. To keep this error source under control one thus has to be able to determine the solar radiation pressure accurately enough such that corrective calculations can be performed. Alternatively, one must wait for drag-free satellites that can perform at least one order of magnitude better than current technology.

The gravitational fields of the Moon and the Sun cause relative accelerations between the Earth and the orbiting clocks. The amplitudes of these accelerations are of the order of $10^{-7} \mathrm{~g}$ (Moon) and $10^{-8} \mathrm{~g}$ (Sun). The influence of the other planets of the solar system plays only a minor role. For Jupiter, e.g., we obtain an influence of the order of 
$10^{-12} \mathrm{~g}$. The positions of the Moon and the Sun are known with much higher accuracy than is needed to determine their gravitational field at the level of $10^{-11} \mathrm{~g}$; therefore, in principle, the influence of the Moon and the Sun can be properly taken into account [30].

It should be remembered that we have provided analytic expressions for $\tau_{ \pm}$only for equatorial circular orbits and for spherical orbits with definite inclination. Our treatment must therefore be extended to eccentric orbits. This will probably require numerical integration of the corresponding geodesic equations. One should keep in mind that even initially circular orbits will acquire eccentricity over time due to the perturbative accelerations mentioned above. It is expected from these considerations and the previous discussion that the measurement of this gravitomagnetic effect would be similarly complicated as in the GP-B.

Finally it should be remarked that the possibility of sending electromagnetic signals around a rotating mass has been considered by a number of authors [31]. The time difference, according to the observer's clock, for a signal to follow a closed path (by means of "mirrors", etc.) in direct and retrograde directions around a rotating mass is proportional to the gravitomagnetic flux threading the loop. Similar effects arise in the synchronization gap around a rotating mass. One can show that all such effects are smaller than the clock effect considered in this paper by a factor of the order of $\Phi=G M / c^{2} r ; \Phi$ is $<10^{-9}$ for the exterior field of the Earth and $<10^{-6}$ for the exterior field of the Sun [2]. The clock effect is larger than that for light by the inverse of a factor of the order $v^{2} / c^{2} \sim \Phi$; intuitively, the clock accumulates a much larger gravitomagnetic effect since its Keplerian motion has a speed that is much smaller than the speed of light.

\section{Acknowledgments}

Thanks are due to Ralph Metzler for his assistance with the preparation of the figures. Helpful discussions with Adam Helfer, Sergei Kopeikin, and Gerhard Schäfer are gratefully acknowledged. This work has been supported in part by the Alexander von Humboldt Foundation.

\section{Appendix A: Gravitational Larmor Theorem}

The local Larmor equivalence between magnetism and rotation has an exact analog in the theory of gravitation. To elucidate this connection, let us consider the motion of free test particles in the gravitational field given by the metric form (1). The geodesic motion follows from $\delta \int-m c d s=0$; however, for purposes of comparison with the Newtonian equations of motion [32, we consider $\delta \int L d t=0$ with $L=-m c d s / d t$. That is

$$
L=-m c^{2}\left[1-2 \Phi-(1+2 \Phi) \frac{v^{2}}{c^{2}}+\frac{4}{c^{3}} \mathbf{v} \cdot \mathbf{A}_{g}\right]^{1 / 2} \text {. }
$$


It follows from this Lagrangian that the canonical momentum is given by

$$
\mathbf{P}=m \Gamma(1+2 \Phi) \frac{d \mathbf{x}}{d t}-\frac{2 m}{c} \Gamma \mathbf{A}_{g}
$$

where $\Gamma=c d t / d s$ and $\Gamma \approx 1$ for nearly Keplerian motion under consideration here. The analog of equation (A1) in electrodynamics is

$$
L=-m c^{2}\left(1-\frac{v^{2}}{c^{2}}\right)^{\frac{1}{2}}+\frac{q}{c}(-c \phi+\mathbf{v} \cdot \mathbf{A})
$$

where $\phi$ is the electric potential. A perturbative treatment of equation (A1) can bring it to the form of equation (A3) with $q \rightarrow-m$ except for an extra factor of 2 in the term involving the gravitomagnetic vector potential; indeed, the origin of this extra factor is that off-diagonal terms appear in the spacetime interval with a factor of 2 . The simplest way to deal with this situation is to assign a gravitoelectric charge of $-m$ and a gravitomagnetic charge of $-2 m$ to the test particle. In this way, equation (A2) becomes consistent with the standard relationship in electrodynamics between the canonical momentum $\mathbf{P}$ and the kinetic momentum $\mathbf{p}$ of a particle of charge $q$ in an inertial frame in Minkowski spacetime, $\mathbf{P}=\mathbf{p}+q \mathbf{A} / c$. It is convenient to use units such that $G=1$ in what follows. In these units, let us therefore assign gravitomagnetic charges of $q_{B}=-2 m$ to the test particle and $Q_{B}=2 M$ to the central source. The source is rotating; therefore, it has a gravitomagnetic dipole moment $\boldsymbol{\mu}_{g}=Q_{B} \mathbf{J} / 2 M c=\mathbf{J} / c$ and hence a gravitomagnetic vector potential $\mathbf{A}_{g}=\boldsymbol{\mu}_{g} \times \boldsymbol{\rho} / \rho^{3}$. This is consistent with equation (2); hence, the analogy established thus far between magnetism and gravitomagnetism is exact in the approximation under consideration here. That is, the gravitomagnetic dipole moment for a test gyro of spin $\mathbf{S}$ is then $\widehat{\boldsymbol{\mu}}_{g}=-\mathbf{S} / c$ and the rate of precession of this dipole moment in the gravitomagnetic field, $d \mathbf{S} / d t=\widehat{\boldsymbol{\mu}}_{g} \times \mathbf{B}_{g}$, is precisely given by equation (3). Moreover, the comparison of equation (A1) with its electrodynamic analog (A3) leads to the interpretation that the factor of 2 in the gravitomagnetic charge has to do with the fact that $d s^{2}$ is a quadratic form or, equivalently, that gravitation is a spin-2 field.

The correspondence between magnetism and gravitomagnetism can be simply extended to the Larmor theorem. Thus the gravitomagnetic Larmor frequency is given by $\Omega_{L}=$ $q \mathbf{B}_{g} / 2 m c=-\mathbf{B}_{g} / c=-\boldsymbol{\Omega}_{P}$. The gravitational Larmor theorem obviously holds for the gravitomagnetic precession of a gyroscope, since it means that the same rate of precession would be obtained locally for a free ideal gyroscope in the absence of gravitation but observed from a frame rotating with frequency $\Omega_{L}=-\Omega_{P}$. Further applications of these ideas are contained in [18].

In view of the intrinsic significance of the gravitational Larmor theorem and its basic relationship with Einstein's principle of equivalence, it is important to point out some of its underlying features. The theorem is formulated with respect to preferred standard observers in the background Cartesian coordinate system, i.e. the rest frame of the center of mass of the rotating source. Furthermore, it has been assumed in our discussion thus far that gravitomagnetism arises from mass currents, as in the case of a rotating planet 
or star, so that the gyromagnetic ratio is then unity. In this case, $q_{E} \neq q_{B}$ and hence Lorentz invariance is broken. It is necessary to point out, however, that our treatment can be extended to the gravitomagnetic field of a rotating black hole as well.

Consider first a test gyro in the field of an arbitrary rotating configuration. It is reasonable to suppose that the gravitomagnetic field is independent of the specific nature of the source in the linear approximation under consideration here. Hence, let $\boldsymbol{\mu}_{g}=\kappa \mathbf{J} / c$ for the source and $\widehat{\boldsymbol{\mu}}_{g}=-\kappa \mathbf{S} / c$ for the test gyro, where $\kappa$ is a universal constant regardless of the nature of the rotating systems. Then, $d \mathbf{S} / d t=\widehat{\boldsymbol{\mu}}_{g} \times \mathbf{B}_{g}$ implies that $\kappa^{2}=1$ and we choose $\kappa=1$ by convention and without any loss in generality. Next, let $\mu=\gamma Q J / 2 M c$, where $\gamma$ is the gyromagnetic ratio and $\kappa=\gamma Q_{B} / 2 M=1$. In view of our previous arguments, cf. equations (A1)-(A3), $Q_{B}=2 M$ and $\gamma=1$; however, in the absence of such arguments the simplest possibility is $Q_{E}=Q_{B}=M$ and hence $\gamma=2$. In fact, the charged Kerr system has $\mu=Q a$ and $\gamma=2$. In this way, the gravitational Larmor theorem can be extended to the exterior field of a Kerr system. Our results suggest, but do not prove, that $\gamma=2$ for the Kerr-Newman system since gravity is a spin-2 field. That is, the factor of 2 in the gravitomagnetic charge of a rotating mass that is connected with the spin- 2 character of the linearized gravitational field simply goes over to the gyromagnetic ratio for the case of a charged Kerr configuration. This apparent independence of $\gamma$ from the detailed electromagnetic nature of the source for this case is supported by the circumstance that $\gamma=2$ extends to the generalized Kerr-Newman spacetime [33], which contains an infinite set of multipole moments, indicating the insensitivity of the gyromagnetic ratio to the detailed structure of the source. Further evidence for this viewpoint comes from the fact that a proper classical interior electromagnetic source for the exterior Kerr-Newman field should have $\gamma=1$ in the absence of gravity. In fact, the gyromagnetic ratio of the Kerr-Newman system loses its meaning once one isolates the electromagnetic source of the Kerr-Newman geometry by simply turning the gravitational interaction off; that is, one is then left with only a charge $Q$ and a static magnetic dipole moment $Q a$.

\section{Appendix B: Gravitational Holonomy}

In general relativity the local geometry of spacetime is determined by a metric $g$. Parallel transport in spacetime is then described by means of a Riemannian connection one-form \{\} $\Gamma_{\alpha}^{\beta}$ which is derived from the metric $g$. The prescription of parallel transport is the following: Consider a linear frame $e_{\alpha}$ at a point $x$ of spacetime. Parallel transport of $e_{\alpha}(x)$ to an infinitesimally neighboring point $x^{\prime}=x+d x$ is defined by the identification of the frame

$$
\left(e_{\alpha}+d e_{\alpha}\right)\left(x^{\prime}\right)=e_{\alpha}^{\prime}+d e_{\alpha}\left(x^{\prime}\right):=e_{\alpha}^{\prime}+\stackrel{\{\}}{\Gamma}_{\alpha}^{\beta}\left(x^{\prime}\right) e_{\beta}^{\prime}
$$


with the parallel transported frame $e_{\alpha}(x)$. I It is well known that this Riemannian parallel transport is both metric preserving and torsion-free. The Riemannian curvature twoform

$$
\stackrel{\{\}}{R}_{\alpha}^{\beta}:=d \stackrel{\{\}}{\Gamma}_{\alpha}^{\beta}-\stackrel{\{\}}{\Gamma}_{\alpha}^{\gamma} \wedge \stackrel{\{\}}{\Gamma}_{\gamma}^{\beta}
$$

is a local measure of the nonintegrability of Riemannian parallel transport. That is, if we parallel transport a frame around an infinitesimal closed loop in spacetime the frame will undergo a "pseudo-orthogonal" linear transformation which is determined by $\stackrel{\{\}}{\alpha}_{\alpha} \beta$. We can generalize this concept and consider parallel transport of a frame around a finite loop. This will again result in a "pseudo-orthogonal" linear transformation of the frame and constitutes the holonomy. More exactly, it is the holonomy of a linear frame bundle with spacetime as base manifold and a Riemannian connection as connection.

More general non-Riemannian spacetime geometries are determined not only by a metric $g$ but also by an independent affine connection which usually can be taken as a Cartan connection $\left(\vartheta^{\alpha}, \Gamma_{\alpha}{ }^{\beta}\right)$ 34. The Cartan connection determines affine parallel transport which generalizes Riemannian parallel transport. While Riemannian parallel transport determines the identification of neighboring linear frames by means of (pseudo-)orthogonal transformations, the affine parallel transport determines the identification of neighboring affine frames by means of affine transformations [35. Note that an affine frame at a point $x$ of a spacetime $M$ is a pair $\left(e_{\alpha}, p\right)(x)$ which consists of a linear frame $e_{\alpha}(x)$ and a point $p(x)$. The affine frame is defined as an element of the affine tangent space $A_{x} M$. We recover from the affine frame $\left(e_{\alpha}, p\right)(x)$ the linear frame $e_{\alpha}(x)$ if and only if $p \in A_{x} M$ is identified with $x$.

The precise prescription of affine parallel transport is the following: Consider a linear frame $e_{\alpha}$ at a point $x$ of spacetime and define $\Gamma^{(T) \alpha}$ as $\Gamma^{(T) \alpha}:=\vartheta^{\alpha}-\delta_{i}^{\alpha} d x^{i}$. Affine parallel transport of $\left(e_{\alpha}, p\right)(x)$ to an infinitesimally neighboring point $x^{\prime}=x+d x$ is defined by the identification of the affine frame

$$
\begin{gathered}
\left(e_{\alpha}+d e_{\alpha}\right)\left(x^{\prime}\right)=e_{\alpha}^{\prime}+d e_{\alpha}\left(x^{\prime}\right):=e^{\prime}{ }_{\alpha}+\Gamma_{\alpha}{ }^{\beta}\left(x^{\prime}\right) e_{\beta}^{\prime} \in A_{x^{\prime}} M \\
(p+d p)\left(x^{\prime}\right)=p^{\prime}+d p\left(x^{\prime}\right):=p^{\prime}+\Gamma^{(T) \alpha}\left(x^{\prime}\right) e^{\prime}{ }_{\alpha} \in A_{x^{\prime}} M
\end{gathered}
$$

with the parallel transported frame $\left(e_{\alpha}, p\right)(x)$. Integration of (B3) and (B4) around an infinitesimal closed loop yields the torsion and curvature two-forms $T^{\alpha}$ and $R_{\alpha}{ }^{\beta}$. The explicit defining formulas are

$$
\begin{array}{cl}
\text { torsion } & T^{\alpha}:=D \vartheta^{\alpha}=d \vartheta^{\alpha}+\Gamma_{\beta}^{\alpha} \wedge \vartheta^{\beta}, \\
\text { curvature } & R_{\alpha}{ }^{\beta}:=d \Gamma_{\alpha}{ }^{\beta}-\Gamma_{\alpha}^{\gamma} \wedge \Gamma_{\gamma}{ }^{\beta} .
\end{array}
$$

Torsion and curvature determine the resulting affine transformation of an affine frame after parallel transport around an infinitesimal closed loop. The torsion measures the

\footnotetext{
${ }^{1}$ This definiton does depend on the choice of $e_{\alpha}^{\prime}=e_{\alpha}\left(x^{\prime}\right)$, of course. Another choice of $e_{\alpha}^{\prime}$ would correspond to another gauge of $\stackrel{\{\}}{\Gamma}{ }_{\alpha}^{\beta}$.
} 
translational part of this transformation while the curvature measures the homogeneous part. Generalizing affine parallel transport to parallel transport around finite loops yields the translational and rotational holonomy which we referred to as gravitational holonomy. It is the holonomy related to an affine frame bundle with spacetime as base manifold and a Cartan connection as connection. Further discussion of gravitational holonomy can be found, for example, in references [36, 37].

\section{References}

[1] See the Proceedings of the Workshop on the Scientific Applications of Clocks in Space, edited by L. Maleki (JPL Publication 97-15, NASA, 1997)

[2] B. Mashhoon, Clocks and General Relativity, in ref. [1], pp. 41-48; J.M. Cohen and B. Mashhoon, Phys. Lett. A 181, 353 (1993).

[3] B. Mashhoon, F.W. Hehl, and D.S. Theiss, Gen. Rel. Grav. 16, 711 (1984).

[4] J.D. North, The Measure of the Universe (Clarendon Press, Oxford, 1965), ch. 3.

[5] E.T. Whittaker, A History of the Theories of Aether and Electricity (Nelson, London, 1951), vol. 1, p.207.

[6] G. Holzmüller, Z. Math. Phys. 15, 69 (1870).

[7] F. Tisserand, Compt. Rend. 75, 760 (1872); 110, 313 (1890).

[8] E. Wiechert, Ann. Phys. (Leipzig) 63, 301 (1920).

[9] F. Hund, Z. Phys. 124, 742 (1948).

[10] D.W. Sciama, Mon. Not. Roy. Astron. Soc. 113, 34 (1953).

[11] W.W. Salisbury and D.H. Menzel, Nature 252, 664 (1974).

[12] B. Mashhoon, J.C. McClune, and H. Quevedo, Phys. Lett. A 231, 47 (1997).

[13] C.W.F. Everitt et al., in: Near Zero: Festschrift for William M. Fairbank, edited by C.W.F. Everitt (Freeman, San Francisco, 1986).

[14] The orbits under consideration in Kerr-Newman spacetime are given by N. Dadhich and P.P. Kale, J. Math. Phys. 18, 1727 (1977). Equation (8) is a generalization of the result given for Kerr spacetime in Cohen and Mashhoon [2]. See also N.V. Mitskevich and I. Pulido Garcia, Sov. Phys. Dokl. 15, 591 (1970) and W. Rindler and V. Perlick, Gen. Rel. Grav. 22, 1067 (1990).

[15] W. Rindler, Phys. Lett. A 233, 25 (1997); Phys. Lett. A 187, 236 (1994).

[16] B. Mashhoon, Found. Phys. 15 (Bergmann Festschrift), 497 (1985); B. Mashhoon and D.S. Theiss, Nuovo Cimento B 106, 545 (1991).

[17] J.J. Sakurai, Phys. Rev. D 21, 2993 (1980) ; E.J. Post, Rev. Mod. Phys. 39, 475 (1967).

[18] B. Mashhoon, Phys. Lett. A 173, 347 (1993).

[19] D.C. Wilkins, Phys. Rev. D 5, 814 (1972).

[20] D.S. Theiss, PhD thesis (Cologne, 1984); Phys. Lett. A 109, 19 (1985); D.S. Theiss et al., MGM: Measurement of the Gravitomagnetic Field of the Earth, Proposal in Response to the European Space Agency Call for Mission Concepts for the Follow-up to Horizon 2000 (1993).

[21] B. Mashhoon and D.S. Theiss, Phys. Rev. Lett. 49, 1542 (1982); Nuovo Cimento B 106, 545 (1991).

[22] M. Nakahara, Geometry, Topology and Physics (Adam Hilger, Bristol, 1990). 
[23] R.J. Petti, Gen. Rel. Grav. 18, 441 (1986).

[24] F.W. Hehl, Found. Phys. 15 (Bergmann Festschrift), 451 (1985).

[25] R.F.C. Vessot and M.W. Levine, Gen. Rel. Grav. 10, 181 (1979).

[26] R.F.C. Vessot, M.W. Levine, E.M. Mattison, E.L. Blomberg, T.E. Hoffmann, G.U. Nystrom, B.F. Farrel, R. Decher, P.B. Eby, C.R. Baughter, J.W. Watts, D.L. Teuber, and F.D. Wills, Phys. Rev. Lett. 45, 2081 (1980).

[27] For informative discussions of the experimental aspects of the clock effect, one of us (B.M.) is grateful to C.W.F. Everitt, M. Kasevich, L. Maleki, J. Prestage, C. Salomon and D.J. Wineland.

[28] S.M. Kopeikin, Sov. Astron. 34, 5 (1990); Manuscripta Geodaetica 16, 301 (1991); V.A. Brumberg and S.M. Kopeikin, Cel. Mech. \& Dyn. Astron. 48, 23 (1990).

[29] P.L. Bender, private communication to F.G. (1997).

[30] One of us (F.G.) wishes to thank P.L. Bender, D.B. DeBra, and G.M. Keiser for enlightening and helpful discussions during the 1997 Alpbach School on Fundamental Physics in Space.

[31] R.W. Davies, in: Experimental Gravitation, edited by B. Bertotti (Academic Press, New York, 1974), p. 405; R.W. Davies and H. Lass, JPL Technical Memorandum no. 58 (1970); J.M. Cohen, A. Rosenblum, and Y. Clifton, Phys. Lett. A 131, 163 (1988); J.M. Cohen, H.E. Moses and A. Rosenblum, Class. Quantum Grav. 1, L57 (1984).

[32] B. Mashhoon, in: Proceedings of the Second Marcel Grossmann Meeting on General Relativity, edited by R. Ruffini (North Holland, New York, 1982), p. 657.

[33] H. Quevedo and B. Mashhoon, Phys. Rev. D 43, 3902 (1991).

[34] É. Cartan, On Manifolds with an Affine Connection and the Theory of General Relativity, English translation of the French original (Bibliopolis, Napoli, 1986).

[35] S. Kobayashi and K. Nomizu, Foundations of Differential Geometry, Vol.I (Interscience, New York, 1963).

[36] J.A. Schouten, Ricci Calculus, 2nd ed. (Springer, Berlin, 1954).

[37] K.S. Stelle and P.C. West, Phys. Rev. D 21, 1466 (1980). 